International Journal of Engineering \&Technology, $7(3.12)(2018) 854-859$
International Journal of Engineering \& Technology
SPC
Website: www.sciencepubco.com/index.php/IJET
Research paper

\title{
Study of Application of Software Defined Network to Real Networks
}

\author{
T.Manoranjitham ${ }^{1}$, K.Sai Vijaya Kumar $^{2}$, B.Varshith ${ }^{3}$ \\ ${ }^{1,2,3}$ Dept of Computer Science, S.R.M University \\ *Corresponding Author Email: ${ }^{1}$ ranjitham69@gmail.com, \\ ${ }_{2}^{2}$ saivijayakumar.kuchipudi@gmail.com, ${ }^{3}$ bavirisettyvarshith@gmail.com
}

\begin{abstract}
This paper presents the application of Software Defined Network to Real Networks. Software Defined Networking is an intriguing concept in the networking and communication industry which provides various uses, from productive network operations to reduced costs in the networking field. The SDN architecture provides the network administrators to implement new network services and easy management of the network. This operation is done by separating the data plane and control plane that makes decision .The data plane forwards packet and control plane manages traffic. In this paper we are studying the application of SDN to Real Networks such as SDN for Internet of Things, Artificial Intelligence, 5G Networks, Wireless Networks.
\end{abstract}

\section{Introduction}

SDN is an efficient approach in networking with productive data traffic within a network.SDN allows network administrators and operators for customization, optimization and virtualization which results in the creation of different type of network services.

In traditional network model control plane and management plane are tightly coupled as shown in figure 1 .Even though this networking model is simple and flexible the network administrators have to configure a huge number of network devices manually and individually, which is quite troublesome. Moving of networking elements such as switch, router or firewall from one place to another requires a lots of change in configurations in the network because the networking software is embedded within the elements itself.

\begin{tabular}{|c|c|}
\hline $\begin{array}{c}\text { Control } \\
\text { Plane }\end{array}$ & $\begin{array}{c}\text { Management } \\
\text { Plane }\end{array}$ \\
\hline \multicolumn{2}{|c|}{$\begin{array}{c}\text { Data } \\
\text { Plane }\end{array}$} \\
\hline
\end{tabular}

Fig. 1: Traditional Network

An intelligent network should posses the ability of dynamic adjustment based on application needed and new properties have to be integrated to the network operations[1].

\section{Networks Using SDN}

SDN basically separates the control and data planes of network elements provides centralized programmability and management

Of networks as shown in figure2.SDN controller instructs networking elements how to handle the data traffic by software messaging. The property of each individual device in the network being individually configured by command-line interface or user interface configured by software. The application layer contains business application with the respective API which requires SDN services by control layer as shown in figure 3 . Control layer is used to provide control functionality to data layer and communicates with it through well defined API. The controller is the network operating system on top of the controller different applications can be done. Switching and forwarding functionalities are done in Infrastructure layer with network switches.

\section{Controller Layer}

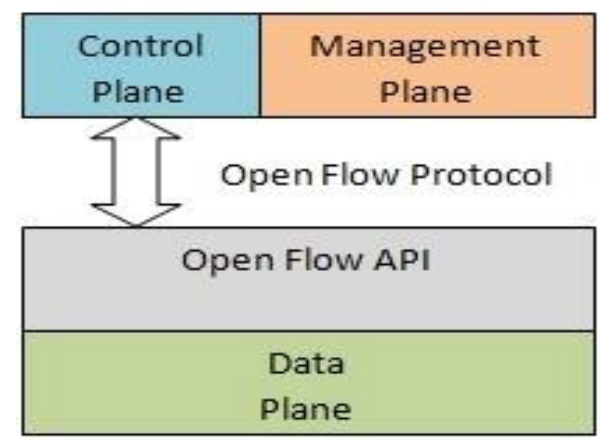

Fig. 2: Seperation of control and data plane

The communication between the control layer and infrastructure layer is done by standard openFlow protocol.All hardware elements that are presented in infrastructure layer must hold OpenFlow protocol. 


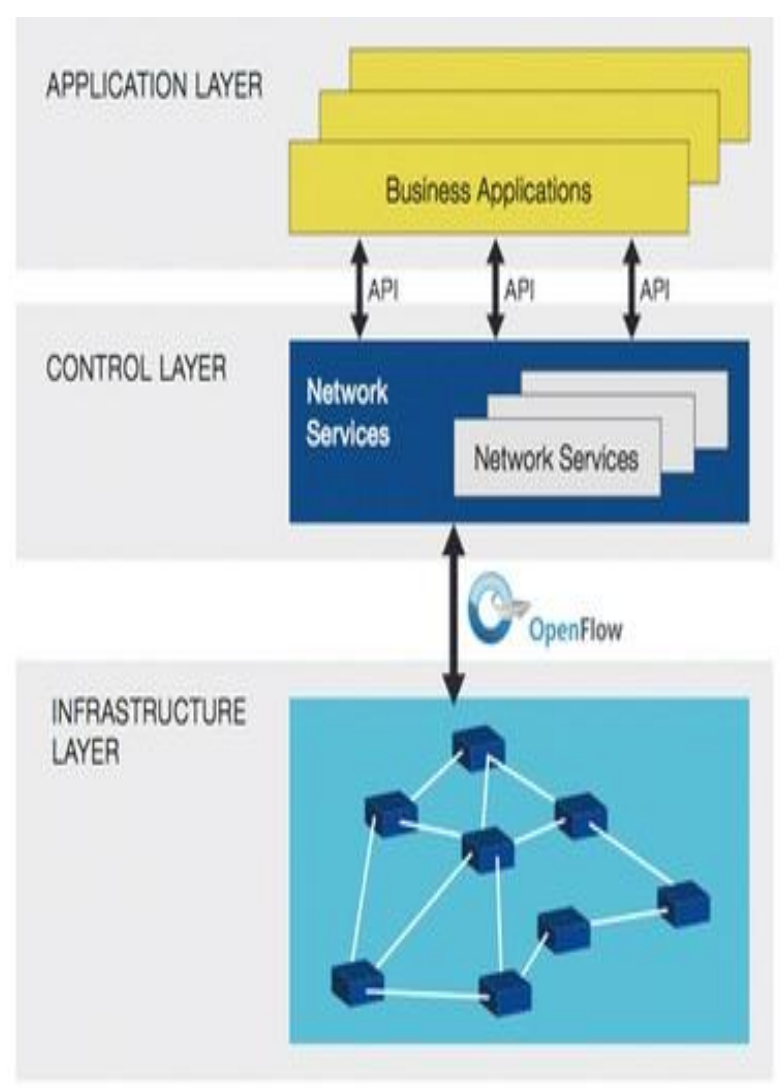

\section{SDN for Internet of Things}

The Internet of Things (IoT) a hot research topic, made innovative ,composite requests to internetworking and networking schemes in future and current networks. In real time, ,networks must encourage different schemes, not just in devices but also in networking behaviour. Furthermore each IoT object has its own design and configuration, to accomplish their respective task. Since the elements in the IoT must be interconnected to the several heterogeneous network and environment where they are working. They need protocols to connect and integrate. Therefore IP for the internet is proposed as solution for IoT. It contains a wide range of network elements, endpoints and the middle of the programmability of elements. The first step toward this massive enterprise is to define the general pattern of separation of control plane and data plane in the switching and routing protocols. This made the intermediate network elements simple, which now behave mere data forwarder. In addition, the program provides insight into the topology and network status in making decisions about the transfer of packets, transmitting the rules on their behalf, and communicating them to the brain of the sender's entity concept set [2].

The first step in designing an SDN and IoT integration is to collect and study the various types of workloads that elements of IoT will transmit in network. Determination of structure and modularity of IoT controllers is the main aspect of design. IoT-responsive high-level controller connects to the SDN controller to interacts with the SDN-enabled networks with the responses of the IoT operation. The architecture is depicted below .

Fig. 3: SDN Architecture

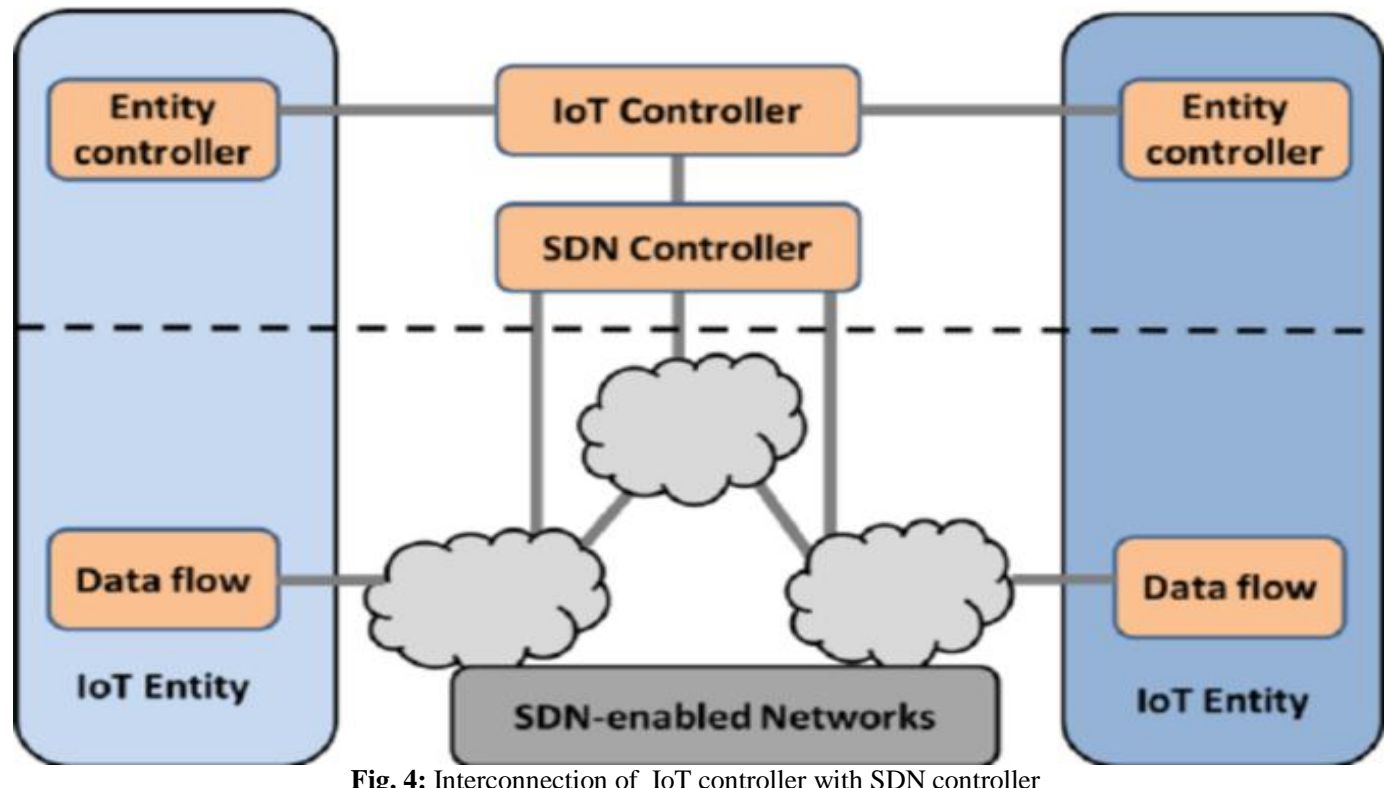

Fig. 4: Interconnection of IoT controller with SDN controller

As shown in figure 4, the overall view of SDN and IoT integration includes the smallest set of functional blocks that are distinguished by modules and their respective planes, objects i.e network plane, data plane or control plane. With the help of internal IoT agent two objects which are attached to the SDN-enabled networks will be able to act together with the IoT controller. The goal is to give the controller with contextual information to make the required decisions and replicate them on the network present. Since the controller is internally modular, new features can be included in IoT without affecting elements in controller.

\section{Challenges and Objectives}

As we discussed the architecture described in the earlier segment reveals a various research questions that often need to be addressed by designing integration mechanisms and architectures. They are briefed as follows

Identification scheme: Common and heterogeneous are the main identification schemes that are present. Objects can use various recognition schemes, mostly relates to the original network protocols they use. In a way to make them interoperable, one need to investigate various identification methods used by IoT network supported protocols. This used to shape the generic map reference which will be used by IoT controller to assign each namespace into a different namespace so that the identifiers used by the object are compatible to its protocol.

Where to instantiate IoT agents: Since IoT agents are lightweight, they are unable to instantiate objects themselves. However, there are many other places where they can instantiate these agents, like the network gateway, the SDN switch, and as a 
different module with IoT controller. These different alternatives from different perspectives in order to incorporate the best programs into the design.

Routing Algorithm: The path between two objects can be found with various routing algorithms. The previous architecture requires that the routing algorithm will consider the condition of two independent but overlapping topologies, SDN and IoT topology. Routing algorithm must think about various aspects such as policy or bandwidth. An appropriate algorithm must be found and evaluated to meet this requirement to decide how it fits the goals of this method.

Forwarding Rule Formulation: After the path calculation is completed, controller must be reflected in various constraints that will be sent to various switches. Although this is simple to do, the working is not straightforward and must take into account the different adjustments, mappings, underlying protocols, appropriate fields and common operations required to accurately transport the packet to its target.

Northbound API Stabilization: The current SDN model includes an API called northbound, used by plug-ins such as IoT controllers .This are used to pass control operations to the SDN controller. However, this API is not quite well defined, so the current approach requires that the stability of this interface to be explored. IoT Controller Modularity: Earlier section, we discussed the major features of IoT controllers, but future enhancements should be opened up as new modules that require in-depth research on the largest and most durable module systems. The

alternative chosen therefore ensures the evolving capabilities of the proposed method.

Deployment Procedure: From the perspective of SDNs and currently available infrastructures, the first deployment procedure should be designed and analyzed first, as this is a key aspect of the proposal's success, validating existing and future networks is very important for the research phase.

Network Security: The SDN approach raise a set of fresh security issues that seem to be one of the biggest problems in SDN. This threats may target the controller by scheduling vulnerabilities, wrong patterns, and Distributed Denial of Service attacks on secure channels.AI and data mining methods used for solving routing problems play a key role in SDN architecture.

\section{SDN Security Attack Vectors}

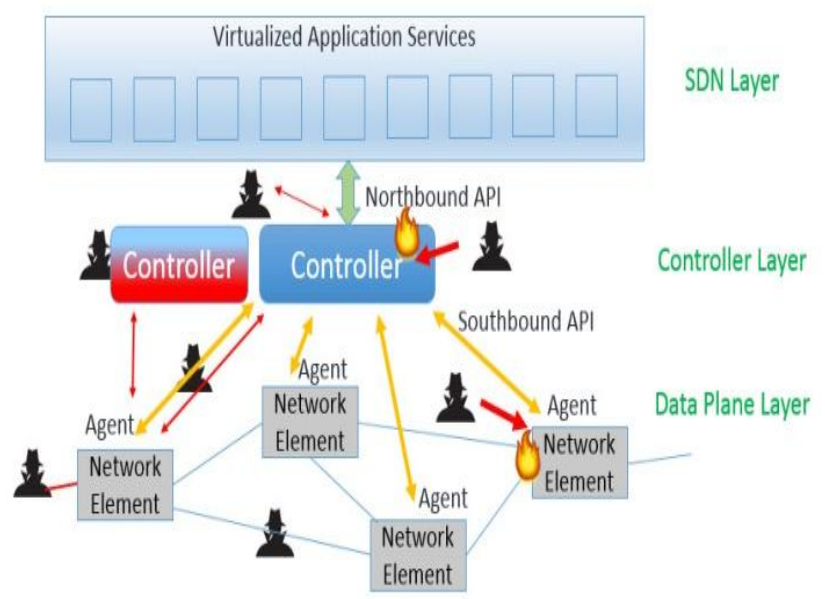

Fig. 5: SDN Security Attack Vectors [12]

By the combination of TRW-CB44 and Rate Limiting information security management is achieved.

\section{Intelligent Network Applications}

Combination of SDN and AI opens the door to building smarter web apps .The controller periodically indicates that the decision maker when to choose a new path .The number of changes is the

\section{SDN for Artificial Intelligence}

Soft computing and AI techniques play an important role in advanced technology, such as robotics, which leads us to improve the performance of current networks. The unification of SDN and AI techniques can tend to adaptive behaviour of networking elements and it helps to develop advanced methods to address traditional network issues and new SDN-related issues.[3]

\section{Load Balancing and Flow Routing}

Load balancing is required to minimize delay and optimize the rate of data transfer of networks which supports multiple routing methods. Load balancing is a defensive method used for some of network attacks such as DDoS attacks. This approach gives an important benefit that is to view and discover the network topology. The neural network's input vector contains the following path information: 1. Bandwidth utilization 2. communication delay 3. Number of hops sent . Data centre load balancing method based on BPN is used internally within the Open vSwitch, reducing the time it takes to send controller routing decisions to the Open vSwitch. Input vectors include Accessible bandwidth and data loss. Genetic Algorithms are located in SDN-based client server architectures. On other hand, an ACO method for QoEsensitive flow routing is proposed $\mathrm{ACO}$ (Ant Colony Optimization) is a group intelligence approach using metaheuristic optimization. In computer networks, Quality of Experience (QoE) means that a customer measures the value of a service from his point of view. The SDN provides user session parameters to the controller, in which ACO algorithm is executed on the weighted graph, here the weight is the delay between vertices and loss rate for each vertices. Fitness function relay on the estimation of the stream type and the corresponding QoE model (ie, audio, video, or data). The maximum QoE obtained by the smallest path increased by $24.1 \%$.

most important parameter used to give the prize. The SDN controller can modify the present path and add layers based on unutilized bandwidth to improve QoE for streaming services. The above routing methods for the shortest path and greedy routes show a drop in frame loss rates of $89 \%$ and $70 \%$ respectively.

\section{SDN for 5G Networks}

Mobile and wireless connectivity have seen phenomenal development over the last few years. These days, 3G / 4G mobile wireless systems are operating to offer connections across IP core networks. This provides endless connectivity with mobile networks such as WLAN, LTE, Bluetooth and 3G . 5G (fifth generation) is based on user-centric concept rather than 3G-centric which is based on operator-centric or 4G-centric servicecentric[4]. The mobile terminal will unite multiple streams from different technologies. $4 \mathrm{G}$ cellular networks have seen multimode mobile terminals. Their goal is to give a single user terminal to work together on various wireless networks to overcome design power and traditional mobile device issues. The Open Wireless Architecture (OWR) is designed to support wireless communication standards across multiple existing wireless radio interfaces and future open architecture platforms. However, growing demand and various modes of Mobile Communications are increasing. For the applications and services purpose, potential $5 \mathrm{G}$ broadband / wireless ,5G will offer a platform for consolidating high volume of network traffic The infrastructure needed for the new equipment provides predictable traffic patterns. 5G wireless network is expected to achieve large-scale development and utilization of functions, as well as complex and powerful large-scale heterogeneous infrastructure connection. Therefore, the network should be able to manage the complex operating environment to support the growing diversity of new and unexpected services and users (Smart City, Mobile Industrial Automation, Vehicle Connectivity, M2M, CCTV, etc.), all of 
which have very different requirements that push the performance and capabilities of mobile networks to the limit. The necessity of intelligence to improve the network of tomorrow in order to successfully deploy and implement a strong wireless world. Software Defined Network (SDN) and Network Function Virtualization (NFV) redefine the network planning to meet the latest demands of new networks. SDN technology has been a good tool for cloud computing operator's vision, which may take part in an essential role in the design of $5 \mathrm{G}$ wireless networks. As a result, future $5 \mathrm{G}$ communications that meet the SDN standard must address the key challenges and requirements for companies, subscribers and operators, giving them more independence to balance operational metrics such as network resiliency and service performance such as Quality of Experience (QoE).

The introduction of $5 \mathrm{G}$ intelligence can solve the problem of network heterogeneity on pointing and giving flexible solutions to solve the complexity of heterogeneous networks (HetNets). Software Defined Networking (SDN) has become a recent intelligent design for network manageability and programmability. The main design of SDN is to disintegrate the data plane and control plane out of the switch and the network operating system is called as controller. SDN provides plain abstract description components, the functionality, the protocols for managing routing plans remotely through secure channels. This idea captures the ordinary forwarding table needs for most switches and their flowcharts. This enables the controller to accomplish network management functions with easy modifications of network behaviour on a centralized control plane. The SDN community employs multiple northbound interfaces (between application and control plane) to provide a higher level of abstraction to program a variety of applications and network-level services. OpenFlow standard has become mainstream technology for the south interface (ie between control plane and network devices) and Rest API as North bound interface.

Intelligent coordination and configuration enable SDN to manage all networked systems. It supports true virtualization networking, high-speed resource allocation, cloud service security and selfservice provisioning. The device itself no longer needs to recognize and handle number of standard protocols, but only accepts directions from the SDN controller.

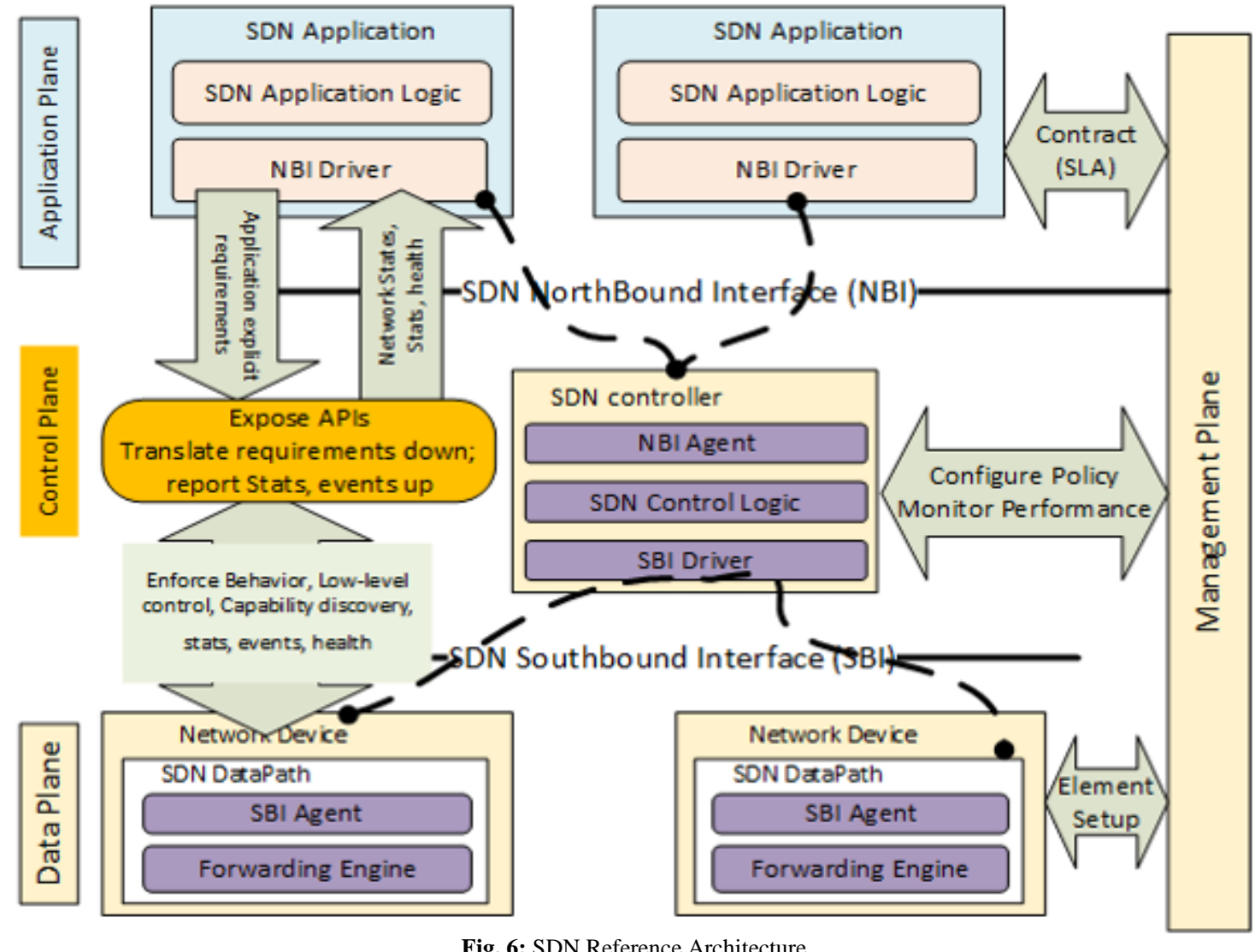

Fig. 6: SDN Reference Architecture

The SDN with application of $5 \mathrm{G}$ wireless network presents new capabilities for network virtualization, automation, and the design of new services on virtualized resources in a secure network. As a result, implementation of data processing and routing capabilities for wireless transportation can be done in software packages in common principle computers and even in the cloud

\section{SDN for Wireless Networks}

The architecture of wireless networks should effectively brace a vast range of services and bring some new ways of considering web application services[4]. The wireless network architecture must support traditional use cases and it should be easy-tointegrate further. In addition, wireless networks manage user mobility while terminals will take a final call between access points in a transparent manner. Mobile devices have the technology that choose and connect to a best access technology and dynamically change the present access technology while ensuring full connectivity.

\section{Mobile Management}

Now current trend is mobility, because use of mobile broadband is continuously growing. By 2020, more than 1,000 times more mobile devices will be covered in the future. These mobile devices have different QOS requirements and will be interconnected to many different and personalized Internet services. Therefore, these developments need to reanalyze the network design, which has led to the benefits of SDN in the ordinary wireless Network scenario. It is essential to know the major problems in this area and how to solve them. Currently, there is no research on SDN Mobility Support till date. Software Defined Wireless Networks provides the SDN technology for wireless networks. The SDWN 
infrastructure can sustain compositing, combining the results of different modules into one set of packet management rules. For example new mobility management protocol should provide to maintain session continuity from the applications and network connections point of view through the dynamic configuration of channels. Multi-reference refers to the end host connected to various networks simultaneously, allowing users to liberally support roaming between wireless infrastructures while supporting providers. This approach will emerge by applying SDN functionality to relay between home and perimeter networks.

\section{Ubiquitous Connectivity}

Being a component of a advance wireless network, end users must communicate with each other, objects surrounding them and machines and sensors. The below figure represents how cellular network will be supplemented by relations with the topology, including M2M complements the different nodes at different levels of user / device and user / device cooperation. Given the variety of interactions with these ubiquitous systems, it will cost more and more network framework that will link new applications and data services such as tablets and smart phones with authoritative multimedia capabilities, and environment, such as roads and even car-to-car exchanges. Therefore, the key design criteria for meeting future $5 \mathrm{G}$ system requirements is ubiquitous system user assets, reduced latency, reliability and energy efficiency, as well as application and system QoS, QoE Heterogeneous service. The SDN prototype can be used as an upper layer of protocol and LR-WPAN. SDN support for LRWPAN extensions is considered impossible because of the networks are greatly controlled in that they need many nodes to cover the extended geographical area and operate on a periodic basis to provide low power, long service long life battery. This approach needs and small software footprint and cross-layer optimization.

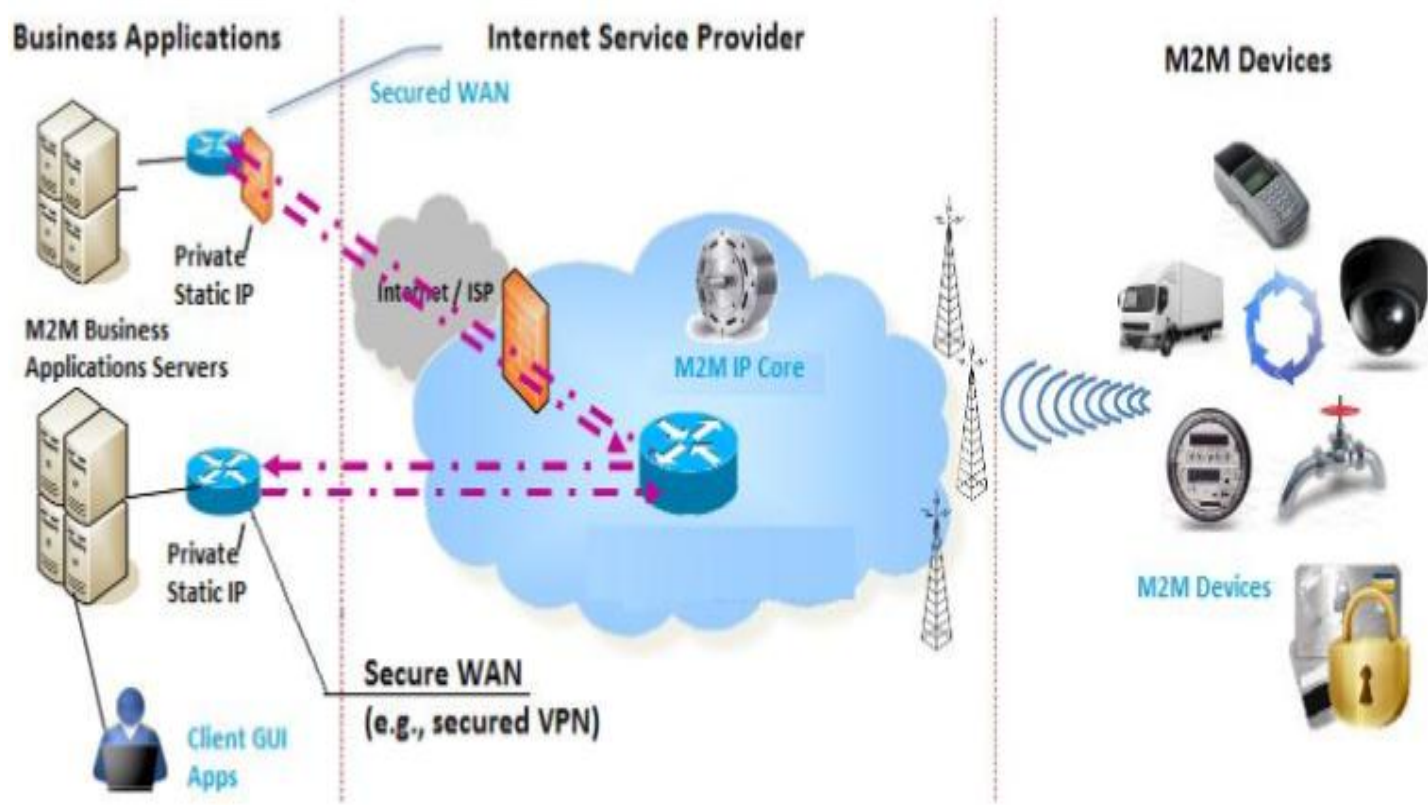

Fig. 7: Integration of ubiquitous systems with $5 \mathrm{G}$ networks [4]

WSDN has the many problem for future SDN networks. The controller must provide the exact prototype to define the rules for the LR-WPAN environment. The WSDN controller must provide comfort to support self-organization, topology discovery, node mobility and auto-configuration. In addition, although energy efficiency is the focus of various studies in the past, there are still problems that the wireless Internet (IoT) will face. IoT ecosystems have become more sophisticated and demanding in terms of performance. The Internet of Things will need new interfaces and protocols. SDNs should greatly decrease the cost of powering the total network, hardware, and running software. For example, a possible solution is to turn off the IoT component while the IoT component is inactive, or to adjust the link rate to minimize the link speed and even consider introducing new routing protocol energy. In the next situation, the SDN controller gathers link usage data for visibility of network traffic and routes traffic according to these protocols.In designing energy-aware protocol should be considered.

\section{Conclusion}

We describe the fundamental role of the basic SDN architecture and the Open Flow protocol and then summarize recent research that provides SDN with a more intelligent network behaviour. Methodology, research in these areas is rapidly evolving and smart hybrid approaches can bring further improvements in the SDN space.

\section{References}

[1] Open Network Foundation - https://www.opennetworking.org Software Defined Networking - O'REILLY

[2] Empowering the Internet of Things with Software Defined Networking [WHITE PAPER] Pedro Martinez-Julia, Antonio F Skarmeta \{pedromj,skarmeta\}@um.es

[3] Majd Latah* and Levent Toker Department of Computer Engineering, Ege University, Bornova, 35100, İzmir, Turkey; latahmajd@gmail.com, toker1960@gmail.com

[4] Leveraging SDN for The 5G Networks: Trends, Prospects and Challenges Akram Hakiri and Pascal Berthou CNRS, LAAS, 7 Avenue du colonel Roche, F-31031 Toulouse, France Univ de Toulouse, UPS, LAAS, F-31031 Toulouse, France

[5] J. Hu and W. Lu, "Open wireless architecture - the core to 4G mobile communications," in Communication Technology Proceedings, 2003. ICCT 2003. International Conference on, 2003.

[6] L. L. Erran, M. Z. Morley and J. Rexford, "CellSDN: SoftwareDefined Cellular Networks," 2012.

[7] M. Wasserman, S. Hartman and D. Zhang, "Security Analysis of the Open Networking Foundation (ONF) OpenFlow Switch Specification," 2012

[8] M. W. S. Hartman and D. Zhang, "Security Requirements in the Software Defined Networking Model," 2013.

[9] Open Networking Foundation. Software-defined networking: The new norm for networks. Available from:https://www. opennetworking.org/images/stories/downloads/sdnresources/whitepapers/wp-sdn-newnorm.pdf.

[10] Kreutz D, Ramos FMV, Verissimo P, Rothenberg CE Azodolmolky S, Uhlig S. Software-defined networking: A 
comprehensive survey. Proceedings of the IEEE. 2015 103(1): 1476

[11] https://www.sdxcentral.com/sdn/definitions/inside-sdn-architecture/

[12] http://www.gtri.com/software-defined-networking-layers-security/

[13] Application of Artificial Intelligence to Software Defined Networking: A Survey

[14] X. Jin, L. E. Li, L. Vanbever and J. Rexford, "SoftCell: scalable and flexible cellular core network architecture.," in CoNEXT, 2013.

[15] L. L. Erran, M. Z. Morley and J. Rexford, "CellSDN: SoftwareDefined Cellular Networks," 2012.

[16] http://www.rfwireless-world.com/Terminology/traditionalnetworking-vs-software-defined-networking.html.

[17] https://www.researchgate.net/figure/IoT-integration-in-SDN47_fig4_287333033 